Efficacy of testosterone nasal gel in patients with seasonal allergies

\title{
Phase 1 pharmacokinetics and phase 3 efficacy of testosterone nasal gel in subjects with seasonal allergies
}

Alan D. Rogol, MD, PhD ${ }^{1}$; Natalia Tkachenko, MD ${ }^{2,4}$; Philipp Badorrek, MD³ Jens M Hohlfeld, $\mathrm{MD}^{3}$; Nathan Bryson, $\mathrm{PhD}^{4}$

${ }^{1}$ University of Virginia, Charlottesville, VA, United States; ${ }^{2}$ Amgen Canada Inc., Mississauga, ON,

Canada; ${ }^{3}$ Fraunhofer Institute for Toxicology and Experimental Medicine (FITEM), Hannover, Germany and

German Centre for Lung Research, Geissen, Germany; ${ }^{4}$ Acerus Pharmaceuticals Corp, Mississauga, ON, Canada

Funding: These studies were sponsored by Acerus Pharmaceuticals SRL (Durants, Christ Church, Barbados).

Cite as: Can Urol Assoc J 2018 March 19; Epub ahead of print. http://dx.doi.org/10.5489/cuaj.4898

Published online March 19, 2018

$* * *$

\section{Abstract}

Introduction: NATESTO ${ }^{\circledR}$ testosterone nasal gel (TNG) is a liquid gel that is applied in the nose for the treatment of male hypogonadism. There is a reasonable concern that administration of TNG to patients with active rhinitis could modify absorption. Results from two clinical studies are reported wherein subjects with allergic rhinitis (AR) subjects are treated with TNG.

Methods: The 24-hour pharmacokinetics (PK) and relative bioavailability of serum total testosterone (sTT) from TNG (11 mg tid ) were determined using a phase 1 Latin-square design with 18 eugonadal AR subjects crossed over between asymptomatic, symptomatic-untreated, and symptomatic-treated (oxymetazoline) conditions. Allergy symptoms, assessed using Total Nasal Symptom Score (TNSS), were induced using grass pollen in an allergy challenge chamber (ACC) prior to administration of TNG. The data are discussed in relation to results from a phase 3 study in 306 hypogonadal patients which compare clinical outcomes of AR and non-AR patients treated with TNG.

Results: PK analysis ( $\mathrm{T}_{\max }$, maximum observed concentration $\left[\mathrm{C}_{\max }\right]$, area under the curve [AUC]) of sTT showed no difference in the rate or extent of absorption of exogenous testosterone from TNG as a function of allergy symptoms. The relative bioavailability also showed all three conditions to be equivalent. However, pre-dose mean STT in AR patients was 21-25\% lower when symptomatic vs. asymptomatic, which is attributed to the allergic reaction. A large phase 3 study, based predominantly on PK measures of sTT, showed that clinical 
outcomes for AR and non-AR patients treated with TNG were identical, including the percentage of patients in the eugonadal range, hormone profiles, and adverse events.

Conclusions: AR does not affect absorption of TNG. Patient outcomes for long-term treatment with TNG for up to one year are not dependent on AR history.

\section{Introduction}

Male hypogonadism is a clinical syndrome that results from a failure of the testes to produce physiologic amounts of testosterone due to disruption at one or more levels of the hypothalamicpituitary-testicular axis. ${ }^{1}$ The syndrome can result in low testosterone levels, clinical symptoms (sexual, somatic and psychological) and impaired spermatogenesis. ${ }^{2}$ There is extensive evidence to support testosterone replacement therapy (TRT) for men with hypogonadism. 1, 3, 4, 5, 6, 7, 8 The intranasal route is increasingly employed for systemic drug delivery because it allows for lower doses due to avoidance of first-pass liver metabolism. ${ }^{9,}{ }^{10}$ TNG is a nasal TRT whose safety and efficacy has been reported. ${ }^{11}$ Allergic rhinitis (AR) is one of the most common adult diseases, affecting between $10 \%$ and $30 \%$ of the population. ${ }^{12,13}$ Nasal inflammation, as might occur with seasonal allergies, as well as decongestant medications to treat AR, could potentially affect absorption of testosterone from TNG. Two clinical studies in AR subjects treated with TNG are reported here. First, a Phase 1 fasting, 3-group/3-period, randomized sequence (i.e. Latin Square) crossover pharmacokinetic study determined the impact of nasal inflammation on the absorption and bioavailability of testosterone from TNG and assessed drug-drug interactions of TNG when symptomatic patients were also treated with a common nasal decongestant, oxymetazoline. An allergy challenge chamber (ACC) was used. ${ }^{14,15}$ Second, results of safety and efficacy from a previously reported Phase 3 study in hypogonadal men were stratified relative to medical history of AR and the two groups were compared. ${ }^{11}$

\section{Methods}

Both clinical studies were conducted in accordance with the Declaration of Helsinki and all applicable laws and regulations in compliance with Good Clinical Practice Guidelines. Each subject signed an informed consent form before initiation of study procedures.

Study drug/s

TNG (4.5\% testosterone) is administered as $5.5 \mathrm{mg}$ testosterone per nostril. ${ }^{16}$ The starting daily dose of TNG is $22 \mathrm{mg} /$ day (11 mg b.i.d) and the maximum daily dose is $33 \mathrm{mg} /$ day (11mg t.i.d). Oxymetazoline hydrochloride $0.05 \%$ is administered as 1 spray per nostril, twice daily, starting 30 min prior to the first dose of TNG and repeated at $12 \mathrm{~h} .{ }^{17}$

Phase 1 study

The Phase 1 PK study was a single site, fasting, randomized, 3-group, 3-period, randomized sequence (Latin Square) crossover design to study the absorption of testosterone from TNG in 
eugonadal men with allergic rhinitis (AR) in 3 test conditions: asymptomatic, symptomatic and symptomatic-treated (Fraunhofer ITEM, Hannover, Germany).

\section{Eligibility}

Eligible subjects were 18-45 years of age with seasonal allergies (positive case history and positive skin prick and/or intradermal test for Dactylis glomerata pollen allergen within 12 months of screening and achieved a Total Nasal Symptom Score (TNSS) of $\geq 6 / 12$ and a congestion score of $\geq 2 / 3$ on at least one card during the 2-hour screening challenge in the ACC). TNSS is a standardized patient-reported questionnaire that measures 4 symptoms (congestion, runny nose, itching and sneezing) on a scale of 0 (none) to 3 (severe). ${ }^{18,19}$ Eligible subjects also had sTT in the normal range, body mass index (BMI) of $\leq 30 \mathrm{~kg} / \mathrm{m}^{2}$, non-smoker for at least 6 months, absence of significant disease or clinical significant abnormal laboratory values, no abnormal otolaryngological findings within 4 weeks of screening and ability to provide informed consent. Exclusion criteria included personal or family history of allergy or hypersensitivity to testosterone or related drugs, past history of anaphylaxis or angioedema, any major illness in the past 3 months or any clinically significant ongoing chronic medical illness (e.g., congestive heart failure, hepatitis, pancreatitis, etc.), prior nasal fractures, subjects with chronic mucosal inflammatory disorders (pemphigus or Sjogren's syndrome, rhinitis sicca), subjects with sinus disease (acute sinusitis, chronic sinusitis, allergic fungal sinusitis), history of nasal surgery (turbinoplasty, septoplasty, rhinoplasty or sinus surgery), and subjects demonstrating a serum prostate specific antigen (PSA) $\geq 4 \mathrm{ng} / \mathrm{mL}$. The full list of exclusion criteria can be found in Supplemental Table 1.

\section{$\underline{\text { Design }}$}

Subjects were randomized in 3 groups 1:1:1 and assigned a different treatment sequence (A, B or C) per Table 1. In each treatment period, subjects received TNG 11mg t.i.d (7am, 1pm, 8pm). A washout period of 4 days allowed endogenous testosterone levels to return to normal, based on known half-life (<100 min). A Symptomatic state was induced with Dactylis glomerata pollen inside an ACC starting $2.5 \mathrm{hr}$ prior to administering each dose of TNG. TNSS scores were also recorded every 15 min for 4 hours after each dose. Subjects were included in the PK set, if all TNSS scores showed absence of symptoms during the entire asymptomatic treatment period ( $\leq 2 / 12$ for the TNSS and $\leq 1 / 3$ for nasal congestion) and demonstrated moderate to severe symptoms during the entire symptomatic periods ( $\geq 6 / 12$ for the TNSS and $\geq 2 / 3$ for nasal congestion). Safety endpoints included tolerability, assessment of adverse events (AEs), nasal examination, and vital signs, which were monitored at each visit. Complete blood count, clinical chemistry profile, and urinalysis were performed at screening and at study completion. All reported AEs were deemed treatment emergent (TEAE). Normal regulatory classification was used to define serious adverse events (SAE) or serious adverse reactions (SAR). 


\section{Phase 3 Study}

Study design and procedures of the multi-site Phase 3 study with 306 hypogonadal males treated with either $22 \mathrm{mg}$ (11mg b.id.) or 33mg (11 mg t.i.d) TNG was reported previously. ${ }^{11}$ The primary endpoint was the percentage of patients in the eugonadal (normal) range, as determined by measurement of sTT levels after 90 days treatment. Two successive, open-label safety extensions allowed patients to remain on study drug to 6 months or to 1 year. Patients with AR were randomized 1:1 to the 2 starting doses in the study. The study was registered with clinicaltrials.gov prior to study start (NCT01446042). Stratification for AR history was performed post-hoc by Nuventra (Raleigh, NC).

Pharmacokinetic analysis

In both clinical studies, blood samples were taken at pre-specified time points over a period of 24hr. Quantification of serum testosterone was performed by LCMS/MS (Range 50-5000 ng/dL, LLOQ 50 ng/dL, CV 2.8-6.2\%) at Analytisch Biologisch Laboratorium (Netherlands). PK parameters were determined using SAS ${ }^{\circledR}$ (SAS Institute, Cary NC, USA) at ICRC-Weyer (Berlin, Germany) for the Phase 1 study and at MedPace (Cincinnati, OH) for the Phase 3 Study. Continuous measurements were summarized by means of descriptive statistics (i.e., number of observations, arithmetic mean, standard deviation [SD], and minimum, median, maximum). Categorical variables were summarized by means of frequency tables (i.e., count and percentages).

Pharmacokinetic analysis in the Phase 1 Study was performed on uncorrected and baseline corrected data. Baseline correction consisted of subtraction of pre-dose sTT from all PK data points. PK parameters were log-transformed before ANOVA using treatment as a factor to calculate LSM ratios and 90\% confidence intervals. Additional details regarding methods are also provided in Rogol et al. ${ }^{11}$

\section{Results}

Phase 1 PK crossover in AR subjects

The demographic characteristics of the 18 AR subjects enrolled in the PK Study are provided in Table 2. All subjects were Caucasian and all groups displayed similar baseline characteristics. Screening of allergic reactivity to Dactylis glomerata to determine eligibility showed mean TNSS scores (SD) of 6.67 (1.81) at $1 \mathrm{hr}$ and 7.56 (2.28) at $2 \mathrm{hr}$.

The 24h PK profiles for all 3 treatment conditions $(\mathrm{N}=14)$ are presented in Fig. 1. The PK parameters $\left(\mathrm{AUC}_{24 \mathrm{~h}}, \mathrm{C}_{\mathrm{avg}}, \mathrm{C}_{\max }\right.$ ) for sTT are presented in Table 3. Four subjects were excluded from the PK set, due to TNSS scores less than 6/12 at any single measure during a symptomatic treatment period. Mean TNSS scores for the PK set $(\mathrm{N}=14)$ in asymptomatic, symptomatic and symptomatic-treated conditions were 0/12 (0), 7.20/12 (1.94), and 6.54/12 (1.92). Sneezing, when symptomatic, ranged from mild to severe (moderate was most common response) as soon as 30min after entering the ACC, prior to administration of the drug product 
and throughout the dosing period. Pre-dose mean sTT (SD) in the asymptomatic state was 536 (106) ng/dL whereas mean sTT was 423 (141) ng/dL and 402 (109) ng/dL in the symptomatic and symptomatic-treated states, respectively. Each PK profile shows 3 discrete peaks, one for each of the three $11 \mathrm{mg}$ doses administered, with mean $\mathrm{T}_{\max }$ (SD) of 53.1(29) min when asymptomatic, 47.8 (32) min when symptomatic and 49.5 (26) min when symptomatic-treated. The ratios and confidence intervals for the relative bioavailability of sTT comparing the 3 treatment conditions were all close to unity (Table 4).

The difference in baseline sTT prior to dosing between asymptomatic and symptomatic states was statistically significant. Further, the mean sTT in the asymptomatic state ranged from 529 to $907 \mathrm{ng} / \mathrm{dL}$, which was consistently higher than observed when symptomatic (370-783 $\mathrm{ng} / \mathrm{dL}$ ) or symptomatic-treated (367-784 ng/dL). The shift in sTT levels is attributed to the allergic reaction directly on endogenous sTT levels. ${ }^{20,21,22}$

To remove the variable contribution of endogenous testosterone, the PK profile was corrected by subtracting the pre-dose total testosterone value from each point in the profile, per Wittert et al. ${ }^{23}$ The resulting baseline-corrected $24 \mathrm{hr}$ PK profile for sTT profile is presented in Fig. 2 and corrected PK parameters are included in Table 3. $\mathrm{C}_{\max }(\mathrm{SD})$ values for the asymptomatic, symptomatic, and the symptomatic-treated states are 527 (176) ng/dL, 487 (151) ng/dL, and 470 (194) ng/dL. Baseline corrected AUC $_{24}$ (SD) values are 3841 (2713) h*ng/dL, 3041 (1967) h*ng/dL, and 3139 (1480) h*ng/dL, respectively. The relative ratios and confidence intervals for $\mathrm{C}_{\mathrm{avg}}, \mathrm{C}_{\max }$ and $\mathrm{AUC}_{24 \mathrm{~h}}$ sTT comparing the asymptomatic and symptomatic condition are all close to 1.0, for uncorrected and baseline corrected PK, indicating no significant difference in relative bioavailability (Table 4).

Reported TEAEs are tabulated in Table 5. All AEs were mild or moderate and resolved without need for intervention. For subjects in symptomatic state, reported TEAEs included respiratory, thoracic, and mediastinal disorders, general disorders and administration site conditions and investigations, including one report each for dysphonia, oropharyngeal pain and allergic rhinitis, injection site phlebitis, nasopharyngitis, and decreased forced expiratory volume. In the symptomatic-treated state, 2 subjects reported dysphonia, while there was one report each of injection site phlebitis (due to sampling), nasopharyngitis, decreased forced expiratory volume, and dizziness. None were deemed related to TNG. Physical examinations, vital signs, and clinical laboratory results did not reveal any clinically significant adverse findings. Overall, TNG was well tolerated.

Post-hoc stratification for AR subjects in phase 3 study A multisite Phase 3 study enrolled 306 hypogonadal men whose demographics were typical of other studies using TRT. ${ }^{11,24,25,26}$ Fifty-eight patients with prior medical history of AR were randomized 1:1 to doses of $11 \mathrm{mg}$ b.i.d (22mg) and $11 \mathrm{mg}$ t.i.d (33mg). During the study, 6/58 (10.3\%) hypogonadal AR patients withdrew consent or were lost to follow-up prior to the primary endpoint assessment at Day 90 compared to 23/247 (9.2\%) of non-AR patients. A total 
of 52 AR patients completed 90 days of treatment, of which 44 continued to 180 days and 10 out to 1 year of treatment.

Baseline characteristics and treatment outcomes for AR and non-AR patients completing the 90-day treatment period are very similar (Table 6). Hypogonadal patients with AR had a mean starting testosterone level of $250 \mathrm{ng} / \mathrm{dL}$ compared to 229 for non-AR patients. After 90 days of treatment, $71.4 \%$ of the AR-patients had testosterone $\mathrm{C}_{\mathrm{avg}}$ in the normal range, whereas $73.7 \%$ of non-AR patients were in the normal range $(p=0.725)$. There was no difference in mean $\mathrm{C}_{\text {avg }}$ or mean $\mathrm{C}_{\max }$ between AR and non-AR patients at 90 days. Mean sTT concentrations (SD) remained elevated for AR patients with values of 672 (484) ng/dL and 581 (394) ng/dL at 180 and 360 days, respectively. Also, 35/44 (80\%) and 8/10 (80\%) of AR patients remained in the eugonadal range at 180 and 360 days, respectively.

The nature of TEAS and incidence were similar between the AR subgroup and non-AR group. The most commonly reported events came from 19 AR patients: nasopharyngitis, 8.2\%; rhinorrhea, 7.8\%; and epistaxis, 6.5\%. The incidence of nasal AEs in AR subjects was either slightly higher or comparable to the full study safety population (i.e., the incidence of epistaxis in the full study Safety Population was 6.5\%, nasal discomfort 5.9\%, nasal congestion 3.9\%, and rhinorrhea 7.8\%). Only 1 subject with AR discontinued treatment as a result of TEAEs. Surprisingly, AR patients reported only a low incidence of seasonal allergy symptoms (3/52 or $5.8 \%$ ) while on TNG for $\geq 6$ months, despite traversing at least 1 high allergy season.

\section{Discussion}

A Phase 1 PK study was designed to determine if there is a significant difference in the absorption of TNG when nasal inflammation is present, using a standardized AR model. ${ }^{14,15,18,19}$ The study combined the use an ACC to induce symptoms. A randomized, 3-group, 3-period, randomized sequence (Latin Square) crossover design was employed to reduce variability in the pharmacokinetic analysis of AR subjects treated with TNG over 3 conditions, when asymptomatic, symptomatic and symptomatic-treated with a nasal decongestant.

The PK profiles are very similar for all 3 conditions showing 3 distinct peaks for each of the 11mg doses administered over each treatment period (Figure 1). However, there is a visible shift (-20\%) of sTT values over the entire PK profile when allergy symptoms are present. The mean calculated sTT parameters, $\mathrm{C}_{\max }, \mathrm{C}_{\min }$ and $\mathrm{C}_{\mathrm{avg}}$, were all somewhat lower for symptomatic conditions versus asymptomatic (Table 3 ). The shift in endogenous sTT observed here could be due to the allergic reaction. Immunological stress in animals and stress in men are both known to decreases endogenous testosterone. ${ }^{20,21,22}$ Seminal work by Chrousos' group suggests a role for cytokines in lowering testosterone levels. ${ }^{27}$

Baseline correction was applied to remove the baseline endogenous testosterone levels, and the immune-mediated shift, as per Wittert. ${ }^{23}$ The baseline corrected PK profiles (Figure 2) and calculated PK parameters (Table 3 and 4) clearly show that sTT absorption of TNG is not affected by the allergy status of the patient nor by the concomitant use of a nasal decongestant. 
A previous study of similar design testing fentanyl nasal spray in AR subjects showed that nasal inflammation alone did not affect absorption of the drug, whereas concomitant use of a decongestant caused a noticeable reduction in $\mathrm{C}_{\max }$ and a significant delay in $\mathrm{T}_{\max } \cdot{ }^{28}$ Such changes were not evident here.

Consistent with this Phase 1 result, study outcomes from a large, multicenter Phase 3 safety and efficacy trial ${ }^{11}$ which included 58 AR patients with ongoing seasonal allergies showed no significant differences in any of the clinical outcome parameters. The pharmacokinetic analyses and primary endpoint (percentage of patients in the eugonadal range) were not affected by a history of AR. Together, these 2 studies support the conclusion that AR history should not be exclusion criteria for consideration of treatment of hypogonadism with TNG.

Notably, 58 AR patients were recruited, 44 patients who completed 6 months, and 10 of whom completed 1 year of treatment, reported only 3 allergy flare-ups were reported. At least 44 of these traversed at least 1 allergy season. This was initially surprising, yet there have been reports that allergy symptoms can be reduced by application of oily formulations in the nose. ${ }^{29,30}$ The formulation here contains castor oil as the primary vehicle, which is spread across the nasal lining with light massaging. This creates a film on the mucosa which may act as a barrier, inhibiting direct contact of air-borne allergens with the mucosa. TNG use may reduce the number and intensity of allergic reactions and could explain these observations.

Limitations in these studies include, the selection of eugonadal male subjects with AR for the Phase 1 study. The use of eugonadal subjects neglects any potential impact of the negative feedback loop within the hypogonadal-pituitary axis, yet should be consistent treatment over all 3 conditions and is expected to yield valid comparative results. The Phase 1 and 3 studies excluded subjects having undergone nasal surgery as well as those with mucosal inflammatory disorders and sinus disease and as such the results presented here are limited to lesser conditions of seasonal and perennial allergies and UTIs.

\section{Conclusions}

The results of a Phase 1 PK study of nasally administered testosterone shows that absorption and relative bioavailability are not significantly affect by allergy symptoms. The use of a decongestant such as oxymetazoline, applied twice-daily as is common clinical practice, did not delay peak absorption nor affect relative bioavailability of testosterone from TNG when compared to the untreated symptomatic state. Although an allergenic model was employed, it is not expected that other causes of acute nasal inflammation, such as an upper respiratory infection, would significantly alter the course of absorption of TNG.

The results presented here did highlight the suppressive effects of allergy on endogenous testosterone levels in eugonadal males. While the results here suggest that this effect does not achieve statistical significance and is unlikely to modify long-term clinical outcomes, it may be of significance when measuring and interpreting testosterone levels in borderline hypogonadal patients. 


\section{References}

1. Bhasin S, Cunningham GR, Hayes FJ, et al. Testosterone therapy in men with androgen deficiency syndromes: an Endocrine Society Clinical Practice Guideline. J Clin Endocrinol Metab 2010; 95:2536-59.

2. Wu FCW, Tajar A, Beynon JM, et al.; for EMAS Group. Identification of late-onset hypogonadism in middle-aged and elderly men. N Engl J Med 2010; 363:123-135.

3. Chiang HS, Cho SL, Lin YC, et al. Testosterone gel monotherapy improves sexual function of hypogonadal men mainly through restoring erection: evaluation by IIEF score. Urology 2009; 73:762-6.

4. Emmelot-Vonk MH, Verhaar HJ, Nakhai Pour HR, et al. Effect of testosterone supplementation on functional mobility, cognition, and other parameters in older men. JAMA 2008; 299:39-52.

5. Reyes-Vallejo L, Lazarou S, Morgentaler A. Subjective sexual response to testosterone replacement therapy based on initial levels of total testosterone. J Sex Med 2007; 4:1757-62.

6. Steidle C, Schwartz S, Jacoby K, et al.; for North American AA2500 T Gel Study Group. AA2500 testosterone gel normalizes androgen levels in aging males with improvements in body composition and sexual function. J Clin Endocrinol Metab 2003; 88:2673-81.

7. Tracz MJ, Sideras K, Boloña ER, et al. Testosterone use in men and its effects on bone health: a systematic review and meta-analysis of randomized placebo-controlled trials. $J$ Clin Endocrinol Metab 2006; 91:2011-16.

8. Wang C, Swerdloff RS, Iranmanesh A, et al.; for Testosterone Gel Study Group. Transdermal testosterone gel improves sexual function, mood, muscle strength, and body composition parameters in hypogonadal men. J Clin Endocrinol Metab 2000; 85:2839-53.

9. Pires A, Fortuna A, Alves G, et al. Intranasal drug delivery: how, why and what for? $J$ Pharm Pharmaceut Sci 2009; 12:288-311.

10. Bitter C, Suter-Zimmermann K, Surber C. Nasal drug delivery in humans. Curr Probl Dermatol 2011; 40:20-35.

11. Rogol AD, Tkachenko N, Bryson N. Natesto ${ }^{\mathrm{TM}}$, a novel testosterone nasal gel, normalizes androgen levels in hypogonadal men. Andrology 2016; 4:46-54.

12. Seidman MD, Gurgel RK, Lin SY, et al.; for Guideline Otolaryngology Development Group. AAO-HNSF. Clinical practice guideline: Allergic rhinitis. Otolaryngol Head Neck Surg. 2015 Feb; S1: 152-43.

13. World Health Organization. White Book on Allergy 2011-2012 Executive Summary. By Prof. Ruby Pawankar, MD, PhD, Prof. Giorgio Walkter Canonica, MD, Prof. Stephen T. Holgate, BSc, MD, DSc, FMed Sci and Prof. Richard F. Lockey, MD. 
Efficacy of testosterone nasal gel in patients with seasonal allergies

14. Krzych-Falta E, Sowa J, Wojas O, et al. Allergen Challenge Chamber: an innovative solution in allergic rhinitis diagnosis. Advances in Dermatology Allergology 2015; 32(6): 421-5.

15. Krug N, Badorrek P, Hohlfeld JM. Experience with an allergen challenge chamber for clinical trials in allergic rhinitis. Clin. \& Experimental Allergy Reviews 2012; Vol 12, S2:1419.

16. Natesto ${ }^{\circledR}$ Product Monograph. Acerus Pharmaceutical Corp. https://pdf.hres.ca/dpd_pm/00036845.PDF Accessed Dec 14, 2016

17. Nasivin ${ }^{\circledR}$ Information for use. Merck https://www.nasivin.de/static/u/pdf/nasivin_nasentropfen.pdf Accessed 2009.

18. Gawchik S, Goldstein S, Penner B, et al. Relief of cough and nasal symptoms associated with allergic rhinitis by mometasone furoate nasal spray. Annals of Allergy Asthma Immunol. 2003; 90: 416-421

19. FDA. Allergic Rhinitis: Developing Drug Products for Treatment. Draft Guidance for Industry 2015.

20. Boonekamp JJ, Ros AH, Verhulst S. Immune activation suppresses plasma testosterone level: a meta-analysis. Biol Letters 2008; 4:741-4

21. Greiner S, Stefanski V, Dehnhard M, et al. Plasma testosterone levels decrease after activation of skin immune system in a free-ranging mammal. General and Comparative Endocrinology 2010; 168: 466-73

22. Viau V. Functional cross-talk between the hypothalamic-pituitary-gonadal and -adrenal axes. J Neuroendocrinol 2002; 14(6):506-513

23. Wittert GA, Harrison RW, Buckley MJ, et al. (2016) An open-label, phase 2, single centre, randomized, crossover design bioequivalence study of AndroForte 5 testosterone cream and Testogel 1\% testosterone gel in hypogonadal men: study LP101. Andrology 2016; 4:41-5

24. Kaufman JM, Miller MG, Garwin JL, et. al. Efficacy and Safety of 1.62 Testosterone Gel for the Treatment of Hypogonadal Men. J. Sex. Med 2011; 8: 2079-89

25. McNicholas TA, Dean JD, Mulder H, et al. A novel testosterone gel formulation normalizes androgen levels in hypogonadal men, with improvements in body composition and sexual function. BJU Int 2003; 91: 69-74

26. Wang C, Ilan N, Arvert S, et al. Efficacy and Safety of the $2 \%$ formulation on testosterone topical solution applied to the axillae in androgen deficient men. Clin. Endorin. 2011; 75: 836-843

27. Tsigos C, Kyrou I, Kassi E, et al. Stress, Endocrine Physiology and Pathophysiology. De Groot LJ, Chrousos G, Dungan K, et al., editors. Endotext [Internet]. South Dartmoth (MA): MDText.com. 2016 March 10 [54 pages] 
28. Perleman M, Fischer AN, Smith A, et al. The impact of allergic rhinitis and its treatment on the pharmacokinetics of nasally administered fentanyl. Int J Clin Pharmacol Ther 2013; 51: 349-356

29. Alsamarai AM, Abdulsatar M, Ahmed Alobaidi AH. Evaluation of topical black seed oil in the treatment of allergic rhinitis. Antiinflamm Antiallergy Agents Med Chem 2014; 13:75-82

30. Andersson M, Greiff L, Wollmer P. Effects of a topical microemulsion in house dust mite allergic rhinitis. Basic Clin Pharmacol Toxicol 2011; 108:146-148.21 


\section{Figures and Tables}

Fig. 1. Linear-scale, arithmetic mean concentration versus time 24-hour pharmacokinetic (PK) curve for serum testosterone in 14 males with allergic rhinitis (AR) under three conditions of allergy challenge. PKs were assessed in the asymptomatic state (Condition 1), as well as symptomatic (Condition 2) or symptomatic and treated with ozymetazoline (Condition 3). The symptomatic allergic state was induced using a Dactylis glomerata pollen challenge within an allergy challenge chamber (ACC). Blood samples were taken at prespecified time points to appropriately characterize individual doses of $11 \mathrm{mg}$ given three-times daily over a period of 24 hours. Testosterone quantification was performed by LCMS/MS. Circle: Condition 1, asymptomatic; Triangle: Condition 2, symptomatic; Square: Condition 3, symptomatic treated.

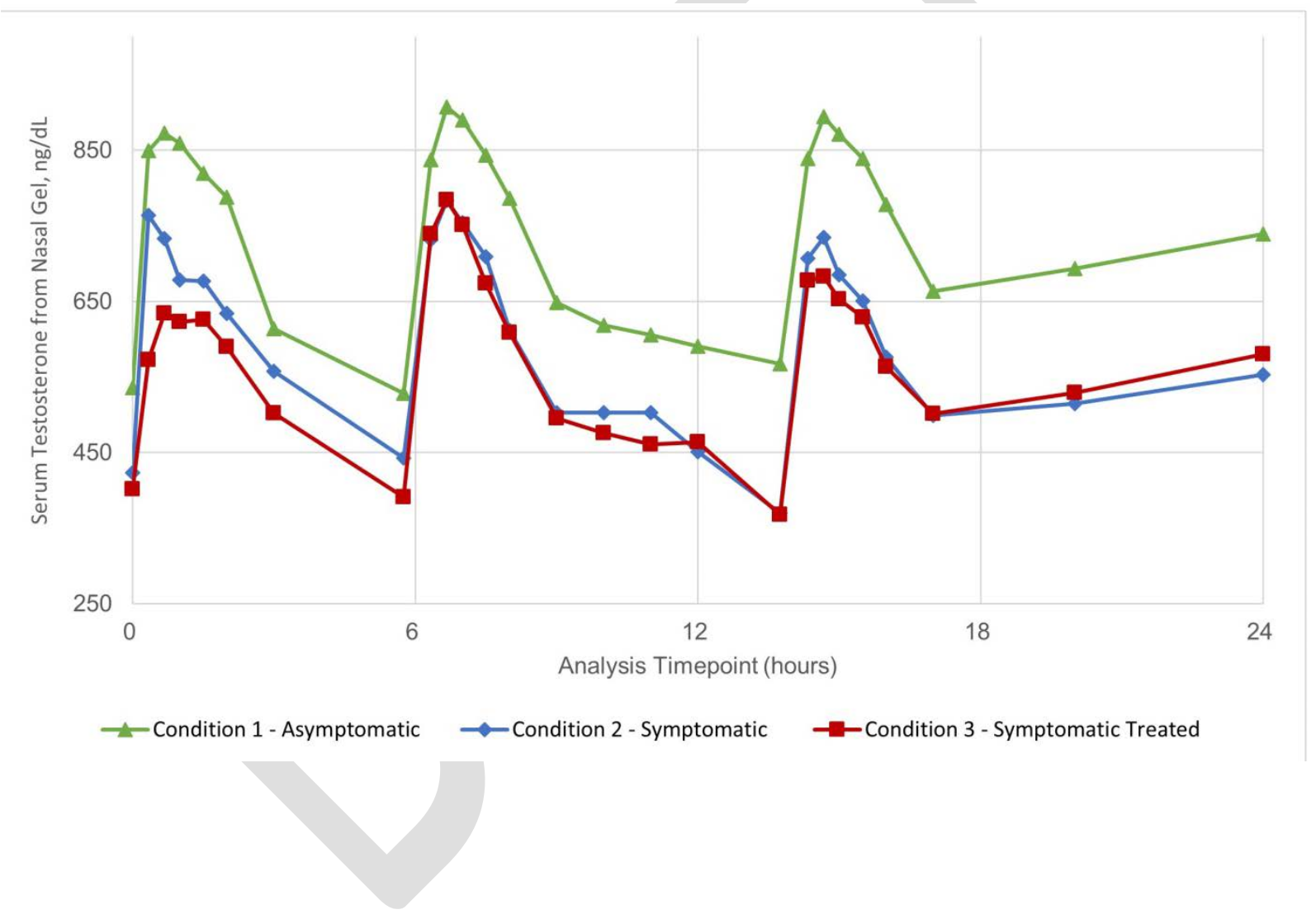


Fig. 2. Linear-scale, arithmetic mean, pre-dose baseline-corrected concentration vs. time 24-hour pharmacokinetic (PK) curve for serum testosterone in 14 males with allergic rhinitis (AR) under three conditions of allergy challenge. PK were assessed in the asymptomatic state (Condition 1), as well as symptomatic (Condition 2) or symptomatic and treated with ozymetazoline (Condition 3). The symptomatic allergic state was induced using a Dactylis glomerata pollen challenge within an allergy challenge chamber (ACC). Blood samples were taken at prespecified time points to appropriately characterize individual doses of $11 \mathrm{mg}$ given three-times daily over a period of 24 hours. Testosterone quantification was performed by LCMS/MS. Baseline corrected PK parameters were performed to relative bioavailability. Circle: Condition 1, asymptomatic; Triangle: Condition 2, symptomatic; Square: Condition 3, symptomatic treated.

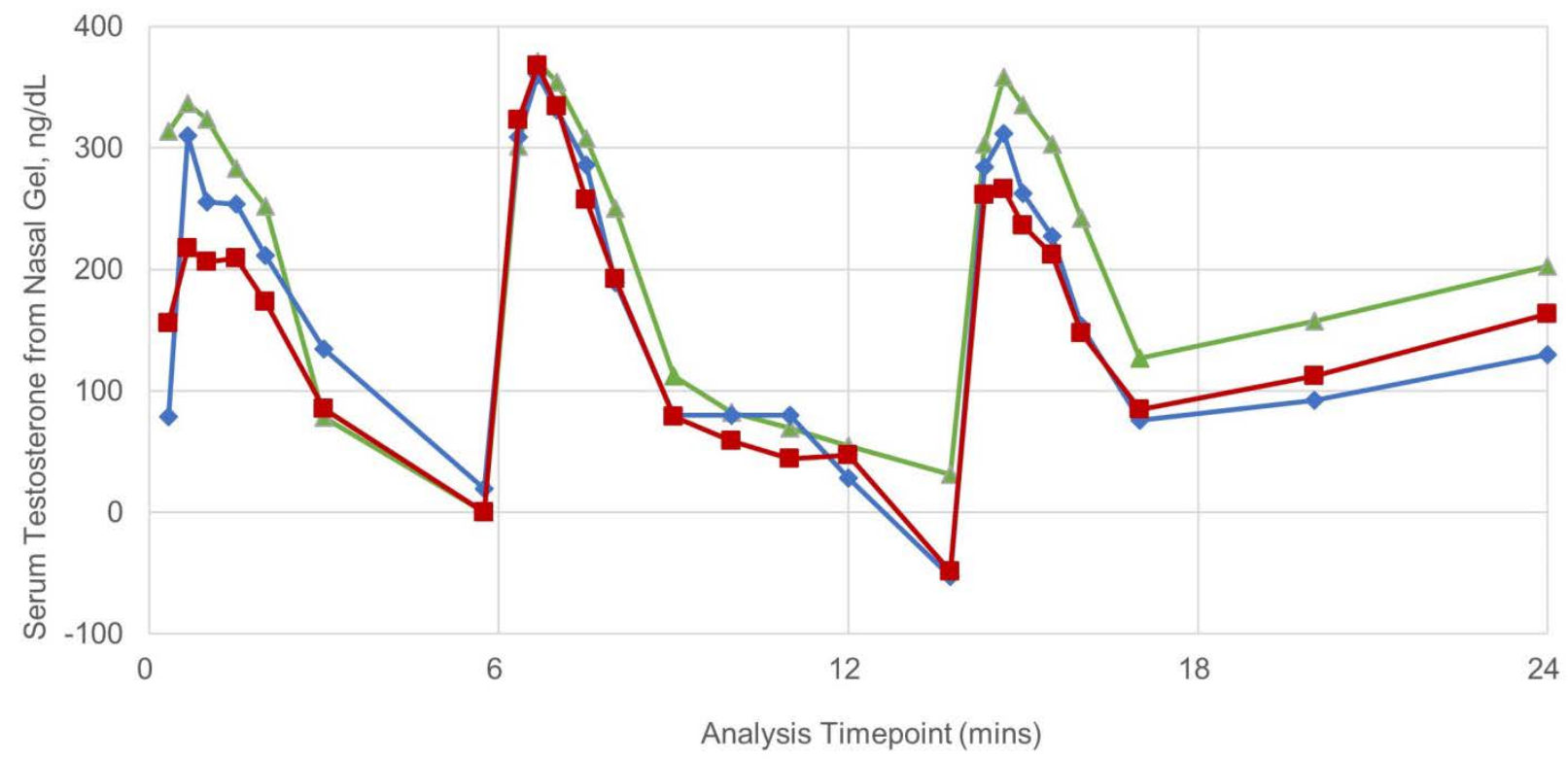




\begin{tabular}{|c|c|c|c|}
\hline & Period 1 & Period 2 & Period 3 \\
\hline Group A & Condition 1 & Condition 2 & Condition 3 \\
\hline Group B & Condition 2 & Condition 3 & Condition 1 \\
\hline Group C & Condition 3 & Condition 1 & Condition 2 \\
\hline
\end{tabular}


Table 2. Baseline demographics in the phase 1 PK study (n=18)

\begin{tabular}{|l|c|c|}
\hline & \multicolumn{2}{|c|}{ Phase 1 } \\
\hline Parameter (mean) & Mean (SD) & Range \\
\hline $\mathrm{n}$ & \multicolumn{2}{|c|}{18} \\
\hline Age (year) & $36(5.8)$ & $27-44$ \\
\hline Height (cm) & $182(6.6)$ & $171-198$ \\
\hline Weight $(\mathrm{kg})$ & $84.9(9.72)$ & $65-103$ \\
\hline BMI (kg/m ${ }^{2}$ ) & $25.4(2.49)$ & $22.2-29.8$ \\
\hline Pre-study, $\mathrm{C}_{\text {max }}$ testosterone ng/dL & $631.4(149.7)$ & $393-848$ \\
\hline Pre-study, $\mathrm{C}_{\text {avg }}$ testosterone ng/dL & $456(92)$ & $305-611$ \\
\hline TNSS in ACC at screening & & \\
1 hour & $6.67(1.81)$ & $4-12$ \\
2 hours & $7.56(2.28)$ & $4-12$ \\
\hline
\end{tabular}

*Average of 2 morning serum testosterone measures. ACC: allergy challenge chamber; BMI: body mass index; $\mathrm{C}_{\mathrm{avg}}$ : average of the observed concentration; $\mathrm{C}_{\max }$ : maximum observed concentration; PK: pharmacokinetics; SD: standard deviation; TNSS: Total Nasal Symptom Score. 
Efficacy of testosterone nasal gel in patients with seasonal allergies

\begin{tabular}{|c|c|c|c|c|c|c|}
\hline & \multicolumn{3}{|c|}{ Uncorrected PK parameters } & \multicolumn{3}{|c|}{ Corrected PK parameters } \\
\hline & Asymptomatic & Symptomatic & $\begin{array}{c}\text { Symptomatic } \\
\text { treated }\end{array}$ & Asymptomatic & Symptomatic & $\begin{array}{c}\text { Symptomatic } \\
\text { treated }\end{array}$ \\
\hline \multicolumn{7}{|l|}{ Mean TNSS } \\
\hline TNSS & $0(0)$ & $7.20(1.94)$ & $6.54(1.92)$ & $0(0)$ & $7.20(1.94)$ & $6.54(1.92)$ \\
\hline \multicolumn{7}{|c|}{ Mean testosterone PK parameters } \\
\hline $\begin{array}{l}\text { Pre-dose } \\
\text { g/dL }\end{array}$ & $534(106)$ & 423(141) & 402 (109) & & _ & - \\
\hline $\mathrm{T}_{\max }, \min$ & $53.1(29)$ & $47.8(32)$ & $49.5(26)$ & - & - & - \\
\hline $\mathrm{C}_{\min }, \mathrm{nd} / \mathrm{dL}$ & $468(94)$ & $344(114)$ & $345(93)$ & - & - & - \\
\hline $\mathrm{C}_{\max }, \mathrm{nd} / \mathrm{dL}$ & $1063(223)$ & $910(242)$ & $872(268)$ & 527 (176) & 487 (151) & $470(194)$ \\
\hline $\begin{array}{l}\text { AUC, ng/dL } \\
h\end{array}$ & $16747(3894)$ & 13217 (3589) & $12778(3380)$ & 3841 (2713) & 3041 (1967) & $3139(1480)$ \\
\hline
\end{tabular}

Negative value ( -50 to $-77 \mathrm{ng} / \mathrm{dL})$ at $\mathrm{t}=13.75 \mathrm{~h}$ in PK profile for all three conditions. AUC: area under the curve; $\mathrm{C}_{\max }{ }^{\prime}$ maximum observed concentration; $\mathrm{C}_{\min }$ : minimum observed concentration; PK: pharmacokinetics; TNSS: Total Nasal Symptom Scores. 


\begin{tabular}{|c|c|c|}
\hline & Asymptomatic/symptomatic & $\begin{array}{c}\text { Symptomatic } \\
\text { untreated/treated with } \\
\text { oxymetazoline }\end{array}$ \\
\hline $\mathrm{n}$ & 14 & 14 \\
\hline \multicolumn{3}{|c|}{ Uncorrected baseline } \\
\hline AUC ratio & $1.03(1.00,1.05)$ & $1.00(0.98,1.02)$ \\
\hline $\mathrm{C}_{\text {avg ratio }}$ & $1.04(1.01,1.07)$ & $1.00(0.97,1.04)$ \\
\hline $\mathrm{C}_{\max }$ ratio & $1.02(0.99,1.06)$ & $1.01(0.98,1.04)$ \\
\hline \multicolumn{3}{|c|}{ Baseline corrected } \\
\hline AUC ratio & $1.03(0.99,1.08)$ & $1.00(0.96,1.05)$ \\
\hline $\mathrm{C}_{\text {avg }}$ ratio & $1.05(0.98,1.13)$ & $1.01(0.93,1.08)$ \\
\hline $\mathrm{C}_{\max }$ ratio & $1.01(0.97,1.05)$ & $1.01(0.97,1.05)$ \\
\hline
\end{tabular}

AUC: area under the curve; CI: confidence interval; PK: pharmacokinetic; TNG: testosterone nasal gel. 


\begin{tabular}{|l|c|c|c|}
\hline \multicolumn{4}{|c|}{ Table 5. Summary of AEs by treatment condition in the phase 1 PK study } \\
\hline Type of event & $\begin{array}{c}\text { Asymptomatic } \\
\text { State }\end{array}$ & $\begin{array}{c}\text { Symptomatic } \\
\text { State }\end{array}$ & $\begin{array}{c}\text { Symptomatic } \\
\text { Treated State }\end{array}$ \\
\hline $\mathrm{n}$ & 18 & 15 & 17 \\
\hline Any AE, n (\%) & $3(16.7)$ & $4(26.7)$ & $5(29.4)$ \\
\hline TEAE, n (\%) & $3(16.7)$ & $4(26.7)$ & $5(29.4)$ \\
\hline AE by intensity, $n$ & & & 4 \\
$\quad$ Mild & 3 & 4 & 2 \\
\hline \multicolumn{1}{|c|}{ Moderate } & 0 & 0 & 4 \\
\hline
\end{tabular}

AE: adverse event; E: number of reported AEs; PK: pharmacokinetic; TEAE: treatmentemergent adverse event. 


\begin{tabular}{|c|c|c|c|}
\hline \multicolumn{4}{|c|}{$\begin{array}{l}\text { Table 6. Phase } 3 \text { study PK results comparing AR HG patients to overall ITT } \\
\text { study population }\end{array}$} \\
\hline & AR patients & Non-AR patients & $\mathbf{p}$ \\
\hline n, completed & 52 & 223 & \\
\hline \multicolumn{4}{|l|}{ Baseline characteristics } \\
\hline Mean age, $\mathrm{y}$ & 55.2 & 54.4 & 0.649 \\
\hline Mean weight, kg & 93.8 & 91.2 & 0.756 \\
\hline Mean BMI & 29.8 & 29.7 & 0.820 \\
\hline Hypogonadism mean duration, $\mathrm{y}$ & 5.7 & 4.5 & 0.135 \\
\hline Etiology, secondary hypogonadism & $86.5 \%$ & $83.9 \%$ & - \\
\hline Mean total testosterone, $\mathrm{nd} / \mathrm{dL}$ & 250 & 229 & 0.098 \\
\hline Mean estradiol at screening & 22.0 & 19.5 & 0.030 \\
\hline Mean DHT at screening & 21.8 & 18.5 & 0.052 \\
\hline Mean FSH & 6.31 & 8.66 & 0.062 \\
\hline Mean LH & 4.36 & 5.81 & 0.023 \\
\hline Mean SHBG & 27.5 & 29.5 & 0.247 \\
\hline \multicolumn{4}{|c|}{ Testosterone levels after 90 days treatment } \\
\hline Mean $\mathrm{C}_{\max }, \mathrm{ng} / \mathrm{dL}$ & 1009 & 978.3 & 0.604 \\
\hline Mean AUC 0-24h, ng h/dL & 8598 & 9283 & 0.054 \\
\hline Mean $\mathrm{C}_{\mathrm{avg}}, \mathrm{ng} / \mathrm{dL}$ & 358 & 387 & 0.054 \\
\hline Mean $\mathrm{C}_{\mathrm{avg}} 22 \mathrm{mg}$ dose, ng/dL & $361(88.4)$ & $378(136)$ & 0.955 \\
\hline Mean $\mathrm{C}_{\mathrm{avg}}$ 33mg dose, ng/dL & $353(89.5)$ & 394 (115) & 0.092 \\
\hline $\mathrm{N}$ in the normal range (\%) & $71.4 \%$ & $73.7 \%$ & - \\
\hline Mean $\mathrm{C}_{\text {avg }}$ estradiol & 25.8 & 25.0 & 0.534 \\
\hline Mean $\mathrm{C}_{\mathrm{avg}} \mathrm{DHT}$ & 31.9 & 35.7 & 0.077 \\
\hline Mean FSH & 3.75 & 5.29 & 0.233 \\
\hline Mean LH & 2.30 & 3.06 & 0.206 \\
\hline Mean SHBG & 23.0 & 24.4 & 0.342 \\
\hline
\end{tabular}

AR: allergic rhinitis; BMI: body mass index; $\mathrm{C}_{\mathrm{avg}}$ : average of the observed concentration; $\mathrm{C}_{\max }$ : maximum observed concentration; $\mathrm{C}_{\min }$ : minimum observed concentration; DHT: dihydrotestosterone; FSH: follicle-stimulating hormone; ITT: intention to treat; LH: luteinizing hormone; SHBG: Sex hormone-binding globulin. 
Efficacy of testosterone nasal gel in patients with seasonal allergies

\section{Supplemental Table 1. Exclusion criteria}

Subjects were entered into the study only if they met none of the following criteria

1. Personal/family history of allergy or hypersensitivity to testosterone or related drugs

2. Past history of anaphylaxis or angioedema

3. Any major illness in the past 3 months or any clinically significant ongoing chronic medical illness (eg, congestive heart failure, hepatitis, pancreatitis, etc.)

4. Presence of any clinically significant abnormal values during screening (eg, significant abnormality of liver function test [LFT], renal (kidney) function test [RFT], etc.)

5 . Hemoglobin $<13 \mathrm{~g} / \mathrm{dL}$ or hematocrit $>52 \%$ during screening

6. Any cardiac, renal, or liver impairment; any other organ or system impairment

7. Asthmatic subjects with a forced expiratory volume in 1 second (FEV1) predicted $<80 \%$

8. History of seizure or clinically significant psychiatric disorders

9. Presence of disease markers for human immunodeficiency virus (HIV) 1 and/or 2, Hepatitis B and/or C (HBsAg and/or HCV) virus

10. History of nasal surgery, specifically turbinoplasty, septoplasty, rhinoplasty ("nose job”), or sinus surgery

11. Subjects with prior nasal fractures

12. Subjects with mucosal inflammatory disorders, specifically pemphigus, or Sjogren’s syndrome, rhinitis sicca

13. Subjects with sinus disease, specifically acute sinusitis, chronic sinusitis, or allergic fungal sinusitis

14. History of nasal disorders (e.g., polyposis, recurrent epistaxis [>1 nose bleed per month]), abuse of nasal decongestants, or sleep apnea

15. Subjects using any form of intranasal medication delivery, specifically nasal corticosteroids and oxymetazoline at the time of screening/enrolment

16. History of asthma and/or ongoing asthma treatment

17. Regular drinkers of more than 3 units of alcohol daily ( 1 unit $=300 \mathrm{~mL}$ beer, 1 glass wine

$=200 \mathrm{~mL}, 1$ measure spirit = $1 \mathrm{cL}$ of pure alcohol), or consumption of alcohol within 48 hours prior to dosing and during the study

18. Subjects demonstrating a positive test for alcohol consumption (using breath alcohol analyzer) at the time of check-in during the admission periods

19. History of, or current evidence of, abuse of alcohol or any drug substance, licit or illicit, before the start of this study

20. Subjects demonstrating a positive test for drugs of abuse in urine (opiates, benzodiazepines, amphetamines, tetrahydrocannabinol [THC], and cocaine) at the time of check-in during admission periods 


\begin{tabular}{|l|}
\hline 21. Inaccessibility of veins in left and right arms \\
\hline 22. Receipt of any prescription drug therapy within 4 weeks of the first admission period \\
\hline $\begin{array}{l}\text { 23. Prescription medication and difficulty in abstaining from over-the-counter (OTC) } \\
\text { medication (except occasional paracetamol/aspirin) for the duration of the study }\end{array}$ \\
\hline 24. Subjects demonstrating serum PSA $\geq 4 \mathrm{ng} / \mathrm{mL}$ \\
\hline $\begin{array}{l}\text { 25. Participation in any other research study involving an investigational new product or } \\
\text { device (IND) or studies that required blood sampling, during the conduct of this study or } 30 \\
\text { days prior to screening of this study (Visit } 1 \text { ) }\end{array}$ \\
$\begin{array}{l}\text { 26. Blood donation (usually } 550 \mathrm{~mL} \text { ) at any time during this study, or within the 12-week } \\
\text { period before study start }\end{array}$ \\
\hline
\end{tabular}

